\title{
RESONANT PHONONS IN ADSORBATES
}

\author{
L. Dobrzynski, B. Djafari Rouhani, P. Zieliński, A. Akjouj, B. Sylla \\ AND E. OUMGHAR
}

\author{
C.N.R.S. - U.S.T.L.(U.R.A. 801), U.F.R. de Physique \\ 59655 Villeneuve d'Ascq CEDEX, France
}

\begin{abstract}
After a brief review on surface phonons, we focus on resonant phonons in adsorbed thin layers. Such resonances may in general be understood within the substrate bulk band as thin layer modes shiften and broaden by the interaction with the substrate phonons. These resonant phonons appear as well-defined peaks in the phonon density of states. Their experimental study started recently by Helium atom scattering spectroscopy. This review will end by a prospective revue done mostly after the presentation of two unpublished results. First the slab resonant phonons may almost be confined in the surface slab even for frequencies falling within the bulk phonon band, if the surface slab is separated from the substrate by a buffer layer. The other prospective unpublished result deals with a model system in which the propagating phonons reach a space point from which they can continue to propagate through two different finite in space channels before reaching again a homogeneous uniform path. Such a system exhibits some frequencies for which the transmission factor vanishes. This phenomenon is related to the resonances associated with the finite additional path offered to the propagation of the phonons.
\end{abstract}

PACS numbers: 68.35.Ja, 68.45.-v

\section{Introduction}

Surface phonons are phonons localized at and propagating along a surface of a condensed medium. Their frequencies fall outside the bulk phonon bands of the bulk medium. So they appear as delta peaks in the phonon density of states, for a given value of their propagation vector $\boldsymbol{k}_{\|}$. The space displacements associated with these vibrations decay exponentially inside the bulk of the medium. Since the prediction of elastic surface waves by lord Rayleigh in 1887, a considerable amount of theoretical, experimental and applied work on surface phonons have appeared. For more information an interested reader can consult many books and review papers, whose references can be traced back from the following ones [1-3]. Most of the works on surface phonons induced by an adsorbed monolayer can also be found starting from these lectures. 
That is why we turn without any further delay to a more recent issue, namely resonant phonons in thin adsorbed layers $[4,5]$. Such resonant phonons appear most often as a continuation within the bulk bands of some phonons confined within the adsorbed layer. Such continuations appear within the corresponding two-dimensional Brillouin zone. In one part of this zone localized phonon branches exist and continue as resonances within another part of this zone. Such resonant phonons are characterized by a peak in the phonon density of states and can be detected experimentally when this peak is sharp enough. Their space displacements are bigger within the adsorbed slab than in the substrate, but at the difference of localized waves they propagate as bulk phonons away from the adsorbed layer.

In the next section we address the case of resonant phonons with a thin adsorbed slab separated from the substrate by another slab called also a buffer layer. In this paper we will not analyse all the properties of such a device, but we will stress only its main property. We found [6] that very sharp resonant phonons may exist in the surface slab for frequencies falling within the substrate bulk and outside the buffer bulk band.

In the final part of this paper, we will present a model system for which resonances may induce transmission zeros for some phonon frequencies [7].

\section{Sharp resonant phonons in a slab separated from its substrate} by a buffer layer

Using the same simple phonon model used before for the study of resonant phonons in a single thin adsorbed slab [5], we investigated the effect of a buffer layer on them. The main finding we report here for the first time can be summarized in a pretty obvious way. When the frequency of a vibrational mode of the adsorbed slab falls outside the bulk band of the buffer layer and inside the substrate bulk band, it may correspond to a very sharp resonance almost completely confined in the adsorbed layer. This existence condition may be fulfilled as well above as below the buffer bulk band [6]. In order to illustrate this general result we used a model (see Fig. 1) of $L(001)$ atomic planes of a simple cubic crystal $(i=1)$ adsorbed on $N-L$ (001) buffer monolayers $(i=2)$ adsorbed on a semi-infinite substrate $(i=3)$. The respective atomic masses and first nearest force constants are $m_{i}$ and $\beta_{i}$ $(i=1,2,3)$. The adsorbed layer-buffer and buffer-substrate force constants are $\beta_{I}$ and $\beta_{I I}$ respectively. For such a simple model, we obtained a semi-analytical expression for the variation of the density of states between the above-defined

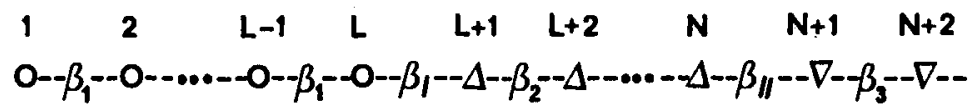

Fig. 1. Two adsorbed slabs of $L$ and $N-L$ monolayers on a semi-infinite substrate. Each point represents an atomic plane. The (001) atomic planes are schematically represented by 0 for the slab (1), $\Delta$ for the buffer slab (2) and by $\nabla$ for the substrate (3). The respective first nearest-neighbor atom interactions are respectively $\beta_{1}, \beta_{2}, \beta_{3}$ within these three materials, $\beta_{I}$ between atoms 1 and 2 and $\beta_{I I}$ between atoms 2 and 3 . 


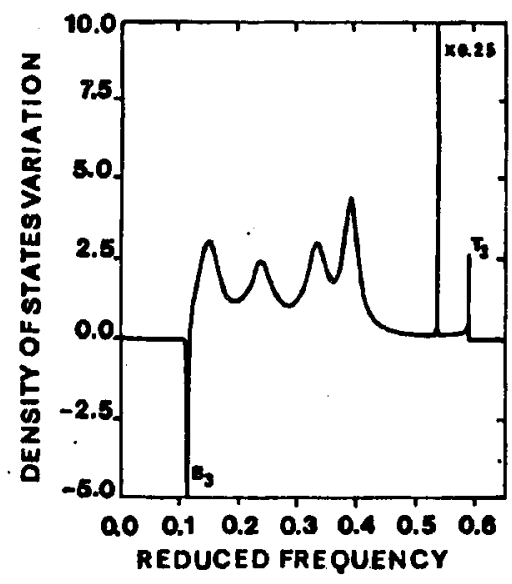

Fig. 2. Variation of the phonon density of states as a function of the reduced frequency (frequency divided by the maximum bulk frequency of the substrate). This variation was defined between a system with two different adsorbed slabs (Fig. 1) and a semi-infinite substrate with the same number of atoms. The parameters used in this application are $L=M=3, m_{1} / m_{3}=0.5, m_{2} / m_{3}=0.75, \beta_{1} / \beta_{3}=1, \beta_{2} / \beta_{3}=0.37, \beta_{I} / \beta_{3}=0.68$, $\beta_{I I} / \beta_{3}=0.5$ and $k_{1} a_{0}=\pi / 8$.

system and a semi-infinite substrate having the same number of atoms. A simple numerical application enables us to obtain the result shown in Fig. 2. The parameters used in this application are $L=M=3, m_{1} / m_{3}=0.5, m_{2} / m_{3}=0.75$, $\beta_{1} / \beta_{3}=1, \beta_{2} / \beta_{3}=0.37, \beta_{I} / \beta_{3}=0.68, \beta_{I I} / \beta_{3}=0.5$ and $k_{1} a_{0}=\pi_{0} / 8$, where $k_{1}$ is the propagation vector along the (100) direction and $a_{0}$ the lattice parameter. The bottom $B_{3}$ and the top $T_{3}$ of the substrate bulk band are indicated in this figure. Starting from the bottom of this band, one may notice first three resonances associated to the three modes of the three layer buffers. The fourth resonance corresponds to the lowest mode of the adsorbed $(i=1)$ slab relatively close to the buffer bulk band. The fifth very sharp resonance corresponds to the second mode of the adsorbed slab. The sixth mode not represented in this figure corresponding to the third mode of the adsorbed slab falls above $T_{3}$ and is completely localized.

When exchanging slabs 1 and 2, similar results can be found below the buffer bulk band, namely in the ultrasonic region. Such sharp waves may prove to be useful for the same kind of electronic devices as those using Rayleigh waves. More information on these resonances relaying on the above-defined model and also within the frame of elasticity theory will appear elsewhere [6]. Our investigations can be compared to those on Brillouin scattering from surface phonons of a $\mathrm{Si} / \mathrm{SiO}_{2}$ bilayer adsorbed on Si, where the authors [8,9] studied acoustic resonances. Their experimental and theoretical approaches may also help to investigate the sharp resonances in an adsorbed slab predicted here outside the bulk band of the buffer slab. 


\section{Transmission resonances and zeros in vibrational waveguides}

The spirit of our investigations already shown above lies in the search of new physical phenomena which may prove to be useful for two- or even one-dimensional devices. After having stressed that the use of buffer layers may provide very sharp resonances in adsorbed thin slabs, we turn now to another new "animal".

In the usual barrier resonant tunneling systems used in quantum waveguides transmission zeros do not occur. It was shown however recently [10] that they may exist in quantum waveguides with resonantly coupled cavities. We found also transmission zeros for phonons within a simple model phonon waveguide.

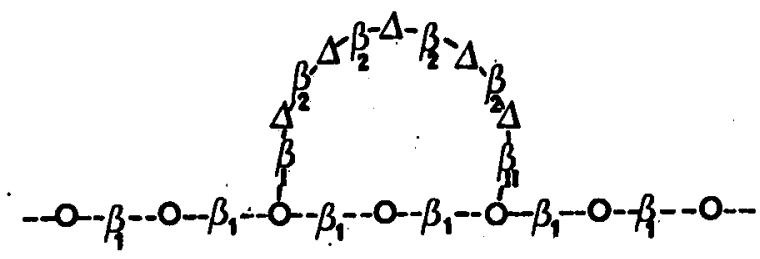

Fig. 3. One monolayer (1) with a coupled resonator (2). Each point represents a row of atoms. The respective first recent neighbor atom interactions are $\beta_{1}, \beta_{2}, \beta_{I}$ and $\beta_{I I}$.

Our model system is presented in Fig. 3. It consists of one simple square monolayer of atoms of mass $m_{1}$ with first nearest-neighbor interaction force constants $\beta_{1}$. This figure presents the projection on the (100) plane of the [010] rows of this monolayer. Between two parallel such rows is established another transmission path through another simple square material whose atomic masses are $m_{2}$ and first nearest-neighbor force constants $\beta_{2}$.

The interactions between the interface atoms connecting the two different materials are respectively $\beta_{I}$ and $\beta_{I I}$. The numbers $L_{1}$ and $L_{2}$ of atomic rows of material 1 and 2, respectively in the two parallel paths shown to be 3 and 5 in this figure can be arbitrary.

Mathematical methods presented fully elsewhere [11] enabled us to find explicit semi-analytical solutions for this model [7].

Without trying to enter here in the analysis of all the physical properties of such a model system, we present in what follows only a single application of the solutions mentioned above. The parameters used in this application are $m_{1} / m_{2}=1, \beta_{2} / \beta_{1}=3, \beta_{I} / \beta_{1}=0.5, \beta_{I}=\beta_{I I}, L_{1}=3, L_{2}=5, k_{2} a_{0}=1.1$, where $a_{0}$ is the lattice parameter.

Figure 4 shows the corresponding transmission factor through the two parallel paths of an incident progressive phonon plane wave, as a function of its reduced frequency. We observe on this curve within the bulk band of material 1 two frequencies for which the transmission factor vanishes. An analysis of the phonon density of states showed [7] that these frequencies correspond to the two well-defined resonances produced by the coupling with the $i=2$ finite transmission path. Two other transmission zeros exist at the bottom and the top of the passing band of the infinite $i=1$ material, respectively and are therefore of no crucial interest for perspective applications. The other aspects and especially the existence of frequencies 


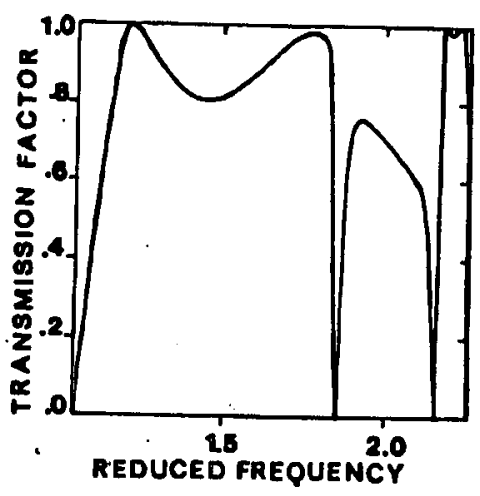

Fig. 4. Transmission factor through the two parallel paths of Fig. 3 as a function of a reduced frequency. The parameters used in this application are $m_{1} / m_{2}=1, \beta_{2} / \beta_{1}=3$, $\beta_{I} / \beta_{1}=0.5, \beta_{I}=\beta_{I I}, L_{1}=3, L_{2}=5$ and $k_{2} a_{0}=1.1$.

for which transmission is not affected by the coupled resonator are common features in double-barrier resonant tunneling systems. We do not then discuss them here.

Let us finally stress that although the above example was presented on a two-dimensional model, it is in fact one-dimensional for a given value of $k_{2}$. In particular in the limit $k_{2}=0$, the model presented in Fig. 4 is one dimensional. The transposition of our phonon model to electrons in the tight building approximation is pretty straightforward [11]. It should then prove to be useful in connection with investigations of devices made out of quantum wires.

\section{Conclusion}

Although this paper deals with localized and resonant phonons in adsorbed thin slabs, its conclusions can be qualitatively transposed to other elementary excitations such as electrons, optical waves, polaritons, spin waves...

Such "animals" as very sharp resonances in thin adsorbed slabs and transmission zeros in systems with coupled resonators can be forseen to exist for any elementary excitation. The discussion of the usefulness of these phenomena for applied devices is however outside our scope. We may only hope that even if, like for Rayleigh waves for example, the applications appear only very late afterwards, our theoretical investigations will contribute to some general understanding and thinking.

\section{References}

[1] A.A. Maradudin, R.F. Wallis, L. Dobrzynski, in: Surface Phonons and Polaritons, in series Handbook of Surfaces and Interfaces, Vol. 3, Ed. L. Dobrzynski, Garland Press, New York 1980.

[2] H. Ibach, Surf. Sci. 299/300, 116 (1994).

[3] G. Benedek, J.P. Toennies, Surf. Sci. 299/300, 587 (1994).

[4] G. Benedek, J. Ellis, A. Reichmuth, P. Ruggerone, H. Schief, J.P. Toennies, Phys. Rev. Lett. 69, 2951 (1992). 
[5] L. Dobrzynski, A. Akjouj, B. Sylla, B. Djafari Rouhani, Acta Phys. Pol. A 81, 85 (1992).

[6] B. Sylla, L. Dobrzynski, B. Djafari Rouhani, A. Akjouj, E.H. El Boudouti, unpublished.

[7] E. Oumghar, P. Zielinski, B. Djafari Rouhani, L. Dobrzynski, unpublished.

[8] C.E. Bottani, G. Ghislotti, P. Mutti, J. Phys., Condens. Matter 6, L85 (1994); G. Ghislotti, C.E. Bottani, Phys. Rev. B 50, 12131 (1994).

[9] F. Nizzoli, C. Byloos, L. Giovannini, C.E. Bottani, G. Ghislotti, P. Mutti, Phys. Rev. B 50, 2027 (1994); Phys. Rev. B 51, 9875 (1995); Phys. Rev. B 51, 9867 (1995).

[10] W. Porod, Zhi-An Shao, C.S. Lent, Appl. Phys. Lelt. 61, 1350 (1992).

[11] A. Akjouj, B. Sylla, L. Dobrzynski, Ann. Phys. (France) 18, 363 (1993). 